\section{3 IMPROVING US NATIONAL DATA ON CHILD MALTREATMENT FATALITIES}

${ }^{1}$ Patricia Schnitzer, ${ }^{2}$ Robert Anderson, ${ }^{2}$ Arialdi Minino, ${ }^{3}$ Theresa Covington. ${ }^{1}$ University of Missouri, USA; ${ }^{2}$ National Centre for Health Statistics, Centres for Disease Control and Prevention, USA; ${ }^{3}$ US National Centre on Fatality Reviews

\subsection{6/injuryprev-2016-042156.283}

Background In the United States (US), national data on child maltreatment $(\mathrm{CM})$ deaths are compiled from state child welfare agencies; approximaly $1600 \mathrm{CM}$ deaths are reported for all 50 states annually. Unfortunately, these child welfare data grossly undercount fatal CM in the US. This project used data from death certificates and child death review (CDR) programs to improve identification and better estimate fatal CM in the US.

Methods CDR data from 9 states with a high proportion of deaths reviewed and recorded in the National CDR Case Reporting System were linked to death certificate/mortality data submitted to the US National Centre for Health Statistics (NCHS). Case inclusion criteria were: children between 7 days and 5 years old that died during 2009 or 2010. A CM classification algorithm that included categories of presumptive, probable, possible CM was developed and applied to the linked deaths. Weights were calculated for each cause of death in each CM category; national CM mortality was estimated by applying these weights to 2011 NCHS mortality data for all 50 states.

Results A total of 10927 deaths meeting the inclusion criteria were identified. Of these, 6660 deaths linked to a record in the NCHS mortality file; 169 additional deaths identified by CDR did not link to a NCHS file record. The NCHS mortality file contained an additional 4098 deaths that did not have a CDR review. A total of 3606 deaths met the definition for presumptive $(\mathrm{n}=907)$, probable $(\mathrm{n}=387)$, or possible $(\mathrm{n}=2312) \mathrm{CM}$ in these 9 states over the 2 study years. This resulted in a national estimate of 1279 presumptive, 500 probable, and 3083 possible $\mathrm{CM}$ deaths for a total of $4862 \mathrm{CM}$ deaths in the US during 2011.

Conclusions It is possible to improve upon the current child welfare-based system of counting fatal child maltreatment in the US by linking CDR and mortality data. In this study, the CDR Program data were essential for confirming child maltreatment in many of the deaths identified.

\section{UNDERSTANDING THE IMPACT OF ADVERSE CHILDHOOD EXPERIENCES ON HEALTH AND WELLBEING IN ENGLAND}

${ }^{1}$ Kat Ford, ${ }^{1}$ Zara Quigg, ${ }^{1}$ Karen Hughes, ${ }^{1}$ Nadia Butler, ${ }^{1,2}$ Mark A Bellis. 'Liverpool John Moores University, United Kingdom; ${ }^{2}$ Public Health Wales, United Kingdom

\subsection{6/injuryprev-2016-042156.284}

Background An emergent body of research is underlining the long-term impacts that adverse childhood experiences (ACEs) have in determining the health and social prospects of individuals. ACEs are now considered one of the strongest predictors of poor health and social outcomes in adults. Individuals with higher numbers of ACEs are found to have higher risks of antisocial behaviour, crime, and violence, and are at increased risk of developing a range of health conditions and ultimately premature mortality.

Methods A household survey was conducted with 5,454 residents aged between 18 and 69 in the United Kingdom in the areas of Northamptonshire, Hertfordshire and Luton during the period June to September 2015. The survey explored childhood experiences before the age of 18, past and current health and social behaviours and outcomes.

Results Four in ten (43.1\%) of all individuals surveyed experienced at least one of the nine ACEs examined. The independent impact of ACEs on health and criminal justice outcomes will be discussed, including the increased risks of harmful behaviours, morbidity and mortality in adulthood from the number of ACEs. The associations between ACEs and socioeconomic markers will be identified. The presentation will provide an overview of the burden of harmful behaviours that would be prevented in the absence of ACEs. Findings across the study areas will be discussed in relation to prevalence of ACEs found in other research within the United Kingdom.

Conclusions The implications of the research findings on interventions to improve health and reduce inequalities will be highlighted. The importance of service planning and effective investment in early years at a local and national level to prevent ACEs and ensure the provision of positive childhood environments for future generations will be addressed.

\section{MARKOV MODEL SIMULATION OF PAEDIATRIC ABUSIVE HEAD TRAUMA PRIMARY PREVENTION AND SCREENING PROGRAMS}

1,2 Marion Bailhache, ${ }^{2,3}$ Antoine Bénard, ${ }^{2,3}$ Louis-Rachid Salmi. ' CHU De Bordeaux, Pole De Pediatrie, France; '2Université De Bordeaux, ISPED, Centre INSERM U897-EpidemiologieBiostatistique, France, ${ }^{3} \mathrm{CHU}$ De Bordeaux, Pole De Sante Publique, Service D'information Medicale, France

\subsection{6/injuryprev-2016-042156.285}

Background Paediatric Abusive Head Trauma (PAHT) is an important cause of morbidity and child-abuse deaths. Several prevention programs exist but their impact is difficult to estimate by lack of knowledge about the beginning of PAHT, difficulties to identify all cases, and the need for large cohorts. We modelled the potential impact of primary prevention and screening programs of PAHT in a hypothetical cohort of 800,000 newborns.

Methods We developed Markov models without intervention, and with primary prevention based on current interventions, screening during repeated consultations, addressed to all families until children are two-years old, and with both programs. Several scenarios were tested regarding potential side effects for parents being mislabeled as abusers (assumed or not to increase the risk of PAHT and decrease participation in screening) and length of the preclinical phase. The time horizon was two years with cycles of fifteen days. Outcomes were number of deaths and abused children avoided. Uncertainty was specified with probability distributions based on literature, or extreme possible values in absence of data.

Results After two years, the median number of death avoided through primary prevention would vary from 6 (CI: 95\%: 2-11) to $28(6-51)$ for 100000 newborns, depending on the probability of PAHT and scenario. Screening could prevent up to $6(0-29)$ deaths or cause up to $66(0-361)$ deaths for 100,000 children born alive. The impact of both programs on mortality and morbidity was uncertain.

Conclusions Our model confirmed the potential benefits of primary prevention and documented the huge uncertainty regarding benefits associated with screening of PAHT. Better estimating the effects of wrongly identifying caregivers as abusers, the evolution of PAHT, and the performance of tests to earlier identify childabuse are necessary. 